\title{
The Characterization of ${ }^{18}$ F-hGTS13 for Molecular Imaging of $\mathrm{x}_{\mathbf{C}}{ }^{-}$Transporter Activity with PET
}

\author{
Corinne Beinat ${ }^{1}$, Gayatri Gowrishankar ${ }^{1}$, Bin Shen ${ }^{1}$, Israt S. Alam ${ }^{1}$, Elise Robinson ${ }^{1}$, Tom Haywood ${ }^{1}$, Chirag B. Patel ${ }^{1,2}$, \\ Emily Carmen Azevedo ${ }^{1}$, Jessa B. Castillo ${ }^{1}$, Ohad Ilovich ${ }^{1}$, Norman Koglin ${ }^{3}$, Heribert Schmitt-Willich ${ }^{3}$, Mathias Berndt ${ }^{3}$, \\ Andre Mueller ${ }^{3}$, Marion Zerna ${ }^{3}$, Ananth Srinivasan ${ }^{1}$, and Sanjiv Sam Gambhir ${ }^{1,4}$ \\ ${ }^{I}$ Department of Radiology, Molecular Imaging Program at Stanford (MIPS), Stanford University School of Medicine, Stanford, \\ California; ${ }^{2}$ Department of Neurology and Neurological Sciences, Stanford University School of Medicine, Stanford, California; \\ ${ }^{3}$ Life Molecular Imaging GmbH (formerly Piramal Imaging) GmbH, Berlin, Germany; and ${ }^{4}$ Departments of Bioengineering and \\ Materials Science and Engineering, Bio-X, Stanford University, Stanford, California
}

\begin{abstract}
The aim of this study was development of an improved PET radiotracer for measuring $\mathrm{x}_{\mathrm{C}}{ }^{-}$activity with increased tumor uptake and reduced uptake in inflammatory cells compared with (S)-4-(3-18 F-fluoropropyl)L-glutamate $\left({ }^{18} \mathrm{~F}-\mathrm{FSPG}\right)$. Methods: A racemic glutamate derivative, ${ }^{18} \mathrm{~F}$-hGTS13, was evaluated in cell culture and animal tumor models. ${ }^{18} \mathrm{~F}-$ hGTS 13 was separated into C5 epimers, and the corresponding ${ }^{18} \mathrm{~F}$ hGTS13-isomer1 and ${ }^{18} \mathrm{~F}-\mathrm{hGTS} 13$-isomer2 were evaluated in $\mathrm{H} 460$ tumor-bearing rats. Preliminary studies investigated the cellular uptake of ${ }^{18} \mathrm{~F}$-hGTS13-isomer2 in multiple immune cell populations and states. Results: ${ }^{18}$ F-hGTS13 demonstrated excellent H460 tumor visualization with high tumor-to-background ratios, confirmed by ex vivo biodistribution studies. Tumor-associated radioactivity was significantly higher for ${ }^{18} \mathrm{~F}$-hGTS13 $(7.5 \pm 0.9$ percentage injected dose $[\% \mathrm{ID}] / \mathrm{g}, n=3)$ than for ${ }^{18} \mathrm{~F}-\mathrm{FSPG}(4.6 \pm 0.7 \% \mathrm{ID} / \mathrm{g}, n=3$, $P=0.01)$. ${ }^{18} \mathrm{~F}-\mathrm{hGTS} 13$-isomer2 exhibited excellent $\mathrm{H} 460$ tumor visualization $(6.3 \pm 1.1 \% \mathrm{ID} / \mathrm{g}, n=3)$ and significantly reduced uptake in multiple immune cell populations relative to ${ }^{18} \mathrm{~F}-\mathrm{FSPG}$. ${ }^{18} \mathrm{~F}-\mathrm{hGTS} 13$-isomer2 exhibited increased liver uptake relative to ${ }^{18} \mathrm{~F}-\mathrm{FSPG}(4.6 \pm 0.8$ vs. $0.7 \pm 0.01 \% \mathrm{ID} / \mathrm{g})$, limiting its application in hepatocellular carcinoma. Conclusion: ${ }^{18} \mathrm{~F}$-hGTS13-isomer2 is a new PET radiotracer for molecular imaging of $\mathrm{x}_{\mathrm{C}}{ }^{-}$activity that may provide information on tumor oxidation states. ${ }^{18} \mathrm{~F}$-hGTS13-isomer2 has potential for clinical translation for imaging cancers of the thorax because of the low background signal in healthy tissue.
\end{abstract}

Key Words: $\mathrm{x}_{\mathrm{C}}{ }^{-}$transporter; oxidative stress; cancer metabolism; PET imaging; ${ }^{18} \mathrm{~F}-\mathrm{hGTS} 13$

J Nucl Med 2019; 60:1812-1817

DOI: 10.2967/jnumed.119.225870

\section{$\mathbf{T}$} he increased uptake of glucose has been exploited clinically to detect tumors and their response to treatment by PET imaging with ${ }^{18} \mathrm{~F}$-FDG $(1,2)$. However, ${ }^{18} \mathrm{~F}$-FDG has limitations, including nonspecificity toward inflammation and high background accumulation in tissues with high glycolytic rates, such as brain and heart (3). To overcome the limitations of ${ }^{18} \mathrm{~F}-\mathrm{FDG}$ and provide additional

Received Jan. 7, 2019; revision accepted May 28, 2019.

For correspondence or reprints contact: Sanjiv Sam Gambhir, Stanford University, 318 Campus Dr., E153, Stanford, CA 94305.

E-mail: sgambhir@stanford.edu

Published online Jun. 6, 2019.

COPYRIGHT (C 2019 by the Society of Nuclear Medicine and Molecular Imaging. information about tumor biology, other fundamental processes in tumor cells would be of utility for detecting cancer, monitoring treatment response, and predicting chemoresistance (4). The enhanced rate of glucose and glutamine uptake to increase cell mass results in elevated levels of oxidative intermediates and subsequent altered redox potential and excess of reactive oxygen species (5). Thiol-containing molecules, including $\mathrm{L}$-cysteine and the tripeptide glutathione (GSH), are key cellular components to neutralize these conditions, and their consumption leads to detoxification of reactive oxygen species and other electrophiles (e.g., chemotherapeutics) (6). GSH, the major thiol-containing endogenous antioxidant, is found in millimolar intracellular concentrations and serves as a redox buffer against various sources of oxidative stress (7-9). A constant supply of GSH and its precursory components is essential for cell survival and provides an advantage for tumor growth. L-cysteine plays a crucial role as a reactive oxygen species scavenger and is also the rate-limiting constituent in GSH biosynthesis (10). L-cysteine is provided to cells via system $\mathrm{x}_{\mathrm{C}}{ }^{-}$, the amino acid transporter that mediates the sodium-independent exchange of extracellular Lcystine and intracellular L-glutamate across the plasma membrane (Fig. 1) (11). Intracellularly, L-cystine is reduced to 2 molecules of L-cysteine, which can be used for GSH biosynthesis. System $\mathrm{x}_{\mathrm{C}}{ }^{-}$is a heterodimeric transporter consisting of 2 subunits: the lightchain $\mathrm{x}_{\mathrm{C}} \mathrm{T}$ (SLC7A11) conferring substrate specificity and the heavychain $4 \mathrm{~F} 2 \mathrm{hc}$ (SLC3A2) (12). It is noteworthy that $\mathrm{x}_{\mathrm{C}}{ }^{-}$is unable to differentiate between its natural substrates L-cystine and L-glutamate for the inward direction of transport (13). The increased activity of $\mathrm{x}_{\mathrm{C}}{ }^{-}$in tumors has previously been exploited by PET imaging with (4S)-4-(3- ${ }^{18}$ F-fluoropropyl)-L-glutamate $\left({ }^{18} \mathrm{~F}-\mathrm{FSPG}\right)$. Pilot clinical studies have been completed examining dosimetry in healthy volunteers and tumor detection in non-small cell lung carcinoma, breast cancer (14), hepatocellular cancer (15), and intracranial malignancies (16). Additional radiotracers, including ${ }^{18} \mathrm{~F}-5$-fluoro-aminosuberic acid, targeting the $\mathrm{x}_{\mathrm{C}}{ }^{-}$transporter have also been described $(17,18)$. Because the $\mathrm{x}_{\mathrm{C}}-$ transporter plays important roles in other, non-tumor-related, diseases, ${ }^{18} \mathrm{~F}-\mathrm{FSPG}$ has high uptake and retention in inflammatory cells (19), including activated T cells and microglia, resulting in the investigation of ${ }^{18} \mathrm{~F}-\mathrm{FSPG}$ to visualize multiple sclerosis (20) and cerebral ischemia (21). With these considerations in mind, second-generation radiotracers for molecular imaging of $\mathrm{x}_{\mathrm{C}}{ }^{-}$were designed (WO/2012/150220), with potential for reduced uptake in inflammatory cells and enhanced tumor visualization. Incorporation of an ultraviolet active moiety was pursued to facilitate radiosynthesis and quality control 


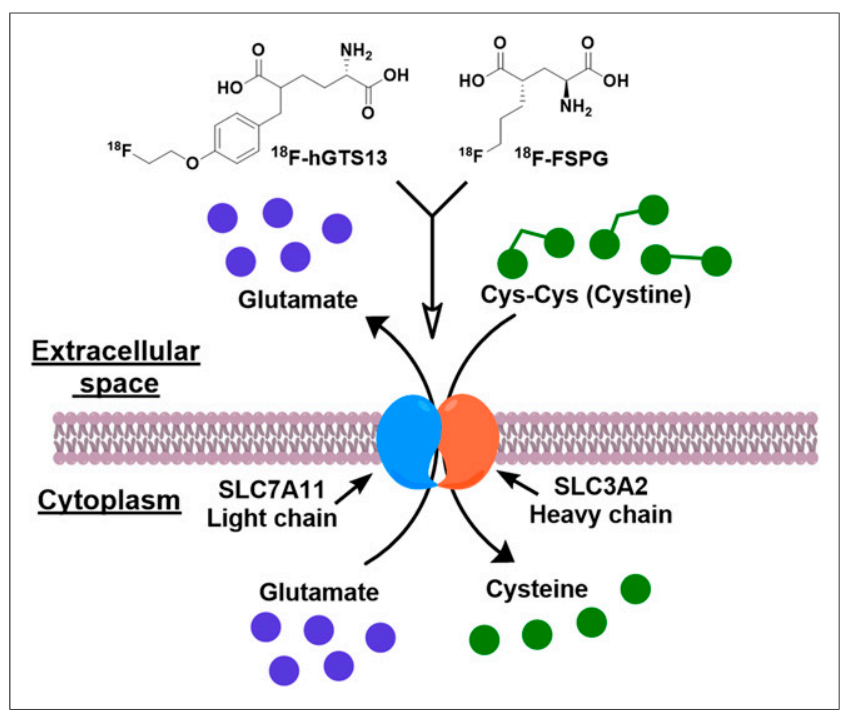

FIGURE 1. Graphical representation of system $\mathrm{x}_{\mathrm{C}}{ }^{-}$, comprising heavy-chain and light-chain $\mathrm{x}_{\mathrm{C}}$ transporter coupled by disulfide bond (S-S). $x_{C}{ }^{-}$imports extracellular cystine in exchange for intracellular glutamate in $1: 1$ ratio. ${ }^{18} \mathrm{~F}-\mathrm{hGTS} 13$ and ${ }^{18} \mathrm{~F}-\mathrm{FSPG}$ bear structural similarities to glutamate and are transported into cell via $\mathrm{x}_{\mathrm{C}}{ }^{-}$.

because ${ }^{18} \mathrm{~F}$-FSPG requires derivatization for quality control analysis at our institution. The compound hGTS13 was selected for further evaluation.

\section{MATERIALS AND METHODS}

\section{General}

Human A549 and H460 tumor cell lines were obtained from ATCC and maintained according to the provider's protocols. Chemicals were obtained from Sigma-Aldrich, Tocris, and Thermo Fisher-Scientific. Synthesis of the precursor, tosylate di-tert-butyl (2S)-2-[(tert-butoxycarbonyl)amino]-5-\{4-[2-(tosyloxy)ethoxy]benzyl\}hexanedioate (compound 1), was adopted from a patent (WO/2012/150220). ${ }^{18}$ F-FSPG was obtained from Stanford Cyclotron and Radiochemistry Facility.

\section{Radiochemistry}

${ }^{18} \mathrm{~F}-\mathrm{h}$ GTS13 was synthesized from nucleophilic displacement of the tosylate group within compound 1 and subsequent deprotection (Supplemental Fig. 1). Full details can be found in the supplemental materials (available at http://jnm.snmjournals.org). The identity of ${ }^{18} \mathrm{~F}-\mathrm{hGTS} 13$ was confirmed through coinjection with reference standard ${ }^{19} \mathrm{~F}-\mathrm{hGTS} 13$ (Supplemental Fig. 2).

\section{Chiral High-Performance Liquid Chromatography}

C5 epimers of compound 1 were separated by high-performance liquid chromatography using a chiral column (Lux 5- $\mu \mathrm{M}$ amylose-1, $150 \times 4.6 \mathrm{~mm})$. An isocratic mobile phase of hexane/isopropanol $(85: 10)+0.1 \%$ diethylamine was used (flow rate, $1.0 \mathrm{~mL} / \mathrm{min}$ ) to give baseline separation of $\mathrm{C} 5$ epimers (retention time, 6.4 and $8.5 \mathrm{~min}$ ).

\section{Cell Uptake Studies}

A549 and H460 cells $\left(2 \times 10^{5}\right)$ were plated into 12 -well plates the day before uptake studies $(n=3$ per condition). On the day of the uptake, prewarmed Hanks balanced salt solution containing about 0.2 $\mathrm{MBq}$ of ${ }^{18} \mathrm{~F}$-hGTS13 was added to individual wells ( $1 \mathrm{~mL}$ per well). Cells were incubated at $37^{\circ} \mathrm{C}$ and $5 \% \mathrm{CO}_{2}$ over a specified time course. For competition studies, wells were pretreated $10 \mathrm{~min}$ before radiotracer addition with the appropriate amino acid or $(S)$-4-carboxyphenylglycine to give a final concentration of $1 \mathrm{mM}(n=3$ replicates per inhibitor). Hanks balanced salt solution containing the inhibitors was aspirated, and fresh Hanks balanced salt solution containing the appropriate inhibitor and $0.2 \mathrm{MBq}$ of ${ }^{18} \mathrm{~F}-\mathrm{hGTS} 13$ was added and incubated at $37^{\circ} \mathrm{C}$ and $5 \% \mathrm{CO}_{2}$ for $60 \mathrm{~min}$. Cells were processed as previously described (22).

\section{$\mathbf{x}_{\mathbf{C}}$ T Small Inhibitory RNA (siRNA)}

A549 cells $\left(6 \times 10^{4}\right)$ were seeded in 12-well plates in antibiotic-free F-12K medium the day before addition of siRNA transfection with lipofectamine 2000 according to the manufacturer's instructions. Specific siRNA sequences targeting the light-chain $\mathrm{x}_{\mathrm{C}} \mathrm{T}$ were produced by Thermo Scientific using the following sequence: CGAGTCTGGGTGGAACTCCTCATAA. Scrambled siRNA (CGAGGTCGGTGTCAACTCCATGTAA) was used as a control. siRNA was added at a final concentration of $25 \mathrm{nM}$, and $\mathrm{x}_{\mathrm{C}} \mathrm{T}$ knockdown was achieved $48 \mathrm{~h}$ after transfection, as confirmed through quantitative reverse-transcription polymerase chain reaction (PCR) and ${ }^{14} \mathrm{C}$-cystine uptake studies. ${ }^{18} \mathrm{~F}$-hGTS13 uptake was measured over $60 \mathrm{~min}$ in cells treated with $\mathrm{x}_{\mathrm{C}} \mathrm{T}$ siRNA and control siRNA at $48 \mathrm{~h}$ after transfection. Cells were processed for tracer uptake studies as described above using ${ }^{18} \mathrm{~F}-\mathrm{hGTS} 13$ or ${ }^{14} \mathrm{C}$-cystine. For ${ }^{14} \mathrm{C}$-cystine measurements, $10 \mathrm{~mL}$ of scintillation liquid were added to lysates and measured using an LS 6500 multipurpose scintillation counter (Beckman Coulter). Untreated cells were used as an additional control, measured $72 \mathrm{~h}$ after seeding.

\section{RNA Isolation, Reverse Transcription, and Quantitative Reverse-Transcription PCR}

Total RNA was extracted with an RNeasy mini kit (Qiagen). cDNA was synthesized with M-MLV reverse transcriptase (Thermo Fisher Scientific) using oligo(dT) primers according to the manufacturer's protocol. Real-time PCR was performed on the iCycler real-time PCR detection system (Bio-Rad) using SYBR green PCR master mix (Life Technologies) with primers specific for $\mathrm{x}_{\mathrm{C}} \mathrm{T}$ and $18 \mathrm{~S}$ ribosomal RNA using annealing and extension temperatures of $56^{\circ} \mathrm{C}$ and $72^{\circ} \mathrm{C}$, respectively. $\mathrm{x}_{\mathrm{C}} \mathrm{T}$ was amplified using primers (forward) $5^{\prime}-$ CAAATGCAGTGGCAGTGACC and (reverse) 5'-AGACAGCAAACACACCACCG, and 18S ribosomal RNA was amplified using primers (forward) 5'-GTAACCCGTTGAACCCCATT and (reverse) 5'-CCATCCAATCGGTAGTAGCG. Transcript levels were normalized to the level of $18 \mathrm{~S}$ ribosomal RNA messenger RNA. The comparative quantitation method $(\Delta \Delta \mathrm{Ct})$ was used to compare the different samples and transform them to absolute values with $2^{-\Delta \Delta \mathrm{Ct}}$ for determining relative fold changes.

\section{Primary Human Immune Cell Isolation, Activation, and Radiotracer Uptake Studies}

Human peripheral blood mononuclear cells were obtained from fresh buffy coat fractions (Stanford Blood Center) using Ficoll-Paque Plus following the manufacturer's instructions (GE Healthcare). T cells, B cells, and monocytes were isolated using a human naive pan $\mathrm{T}$ cell isolation kit, human B cell enrichment kit, and human monocyte isolation kit, respectively (Stemcell Technologies). Isolated cells were subsequently maintained in resting or activated conditions for $2 \mathrm{~d}$. T cells were activated with the T-cell activation/expansion kit using anti-CD3/2/28 coated particles (Miltenyi Biotec). B cells were activated with (S)-4-carboxylphenylglycine oligodeoxynucleotide 2006 ( $1 \mu \mathrm{M}$; Sigma-Aldrich). Monocytes were activated using lipopolysaccharide $(1 \mu \mathrm{g} / \mathrm{mL}$, Escherichia coli O55:B5; Sigma-Aldrich) (23-25). Activation was confirmed by assessing significant changes in morphology in activated versus resting cells.

Radiotracer uptake experiments were performed $48 \mathrm{~h}$ after activation. Cells from the same donor were used to evaluate uptake of both ${ }^{18}$ F-hGTS13-isomer2 and ${ }^{18}$ F-FSPG to minimize donor-dependent variation. T cells, B cells, and monocytes $\left(3 \times 10^{5}\right.$ per well $)$ were prepared in prewarmed Hanks balanced salt solution in a 96-well plate and incubated with about $0.8 \mathrm{MBq}$ of radiotracer per well for $1 \mathrm{~h}$. Cells were processed as previously described (26). 


\section{Animal Studies}

All experiments involving animals were in accordance with protocols approved by the Institutional Animal Care and Use Committee at Stanford University and were performed in accord with the National Institutes of Health Guide for the Care and Use of Laboratory Animals. H460 subcutaneous tumor models were developed as previously described (27). PET/CT imaging of rats was performed with a small-animal hybrid scanner (Inveon; Siemens) and analyzed according to methods previously described by our group (22). Approximately $15 \mathrm{MBq}(400 \mu \mathrm{Ci})$ of each radiotracer was administered to rats intravenously. For comparative radiotracer studies, the same cohort of rats was scanned with each radiotracer; representative images show comparison within the same animal. Static PET scans were acquired $60 \mathrm{~min}$ after injection of radioactivity, and the acquisition of dynamic PET scans commenced immediately before injection of radiotracer. No partial-volume correction was completed.

\section{Ex Vivo Biodistribution Studies}

At completion of PET imaging studies, about 80 min after radiotracer administration, the rats were sacrificed by terminal cardiac puncture and tissues harvested. Radioactivity within individual tissues was determined on a $\gamma$-counter. Radioactivity standards were counted for data normalization. Data were expressed as percentage injected dose (\%ID)/g.

\section{Statistical Analyses}

Data were expressed as mean $\pm \mathrm{SD}$. Statistical significance was determined using a 2-tailed Student $t$ test, with $P$ values of less than 0.05 being considered significant. For analysis across multiple samples, 1- or 2-way ANOVA was used, followed by multiple comparisons of means with Bonferroni adjustment.

\section{RESULTS}

\section{Rapid and Extensive Uptake of ${ }^{18} \mathrm{~F}$-hGTS13 in Cell Culture}

Cell culture studies showed rapid cellular uptake in A549 and H460 tumor cells after addition of ${ }^{18} \mathrm{~F}-\mathrm{hGTS} 13$, with uptake values of $9.7 \% \pm 2.8 \%$ and $21.6 \% \pm 3.6 \%$ in A549 cells and $15.8 \% \pm$ $1.5 \%$ and $32.1 \% \pm 0.8 \%$ in $\mathrm{H} 460$ cells at 30 and $60 \mathrm{~min}$, respectively (Fig. 2A). Strong inhibition of ${ }^{18} \mathrm{~F}$-hGTS13 uptake was evident in the presence of L-glutamate, L-cystine, or the system $\mathrm{x}_{\mathrm{C}}{ }^{-}$-specific inhibitor (S)-4-carboxylphenylglycine. However, no inhibition of uptake was evident in the presence of L-aspartate. The competition profile of ${ }^{18} \mathrm{~F}$-hGTS13 in both A549 and H460 cells is highlighted in Figure $2 \mathrm{~B}$ and indicates the specificity of this radiotracer for system $\mathrm{x}_{\mathrm{C}}{ }^{-}$. We additionally modulated light-chain $\mathrm{x}_{\mathrm{C}} \mathrm{T}$ protein expression in vitro through siRNA; reduced $\mathrm{x}_{\mathrm{C}} \mathrm{T}$ expression was confirmed through PCR and ${ }^{14} \mathrm{C}$-cystine uptake (Supplemental Fig. 3). A significant reduction in ${ }^{18} \mathrm{~F}$-hGTS13 uptake $(78 \%, P<$ $0.0001, n=3$ per condition) was evident in $\mathrm{x}_{\mathrm{C}} \mathrm{T}$ siRNA cells compared with those transfected with control siRNA (Fig. 2C). Competition studies in the presence of L-glutamine, L-leucine, and $\mathrm{L}$-alanine suggest possible involvement of systems $\mathrm{L}$ and $\mathrm{B}^{+0}$ in the uptake of ${ }^{18} \mathrm{~F}$-hGTS13, particularly in A549 cells (Supplemental Fig. 4), whereas additional knockdown studies (Fig. 2C and Supplemental Fig. 5) indicate specificity to system $\mathrm{x}_{\mathrm{C}}{ }^{-}$and a similar specificity profile to ${ }^{18} \mathrm{~F}-\mathrm{FSPG}$.

\section{Evaluation of ${ }^{18} \mathrm{~F}$-hGTS13 in H460 Tumor-Bearing Rats}

In xenograft models of $\mathrm{H} 460$ cells, 10-min static PET/CT imaging was completed $60 \mathrm{~min}$ after injection of ${ }^{18} \mathrm{~F}-\mathrm{hGTS} 13$. The distribution of ${ }^{18} \mathrm{~F}-\mathrm{hGTS} 13$ was characterized by renal clearance and liver uptake (Fig. 3). High tumor uptake $(7.5 \pm 0.9 \% \mathrm{ID} / \mathrm{g})$ was evident at $60 \mathrm{~min}$ after injection of radioactivity. Rats bearing H460 tumors were additionally scanned $1 \mathrm{~d}$ beforehand with ${ }^{18} \mathrm{~F}-\mathrm{FSPG}$ $\mathrm{PET} / \mathrm{CT}$, and levels of radioactivity within $\mathrm{H} 460$ tumors were significantly lower with ${ }^{18} \mathrm{~F}$-FSPG than with ${ }^{18} \mathrm{~F}-\mathrm{hGTS} 13$ (4.6 \pm 0.7 , $P=0.01, n=3)$. Ex vivo biodistribution studies after the ${ }^{18} \mathrm{~F}-$ hGTS13 PET scan corroborated the PET imaging results (Fig. 3C). ${ }^{18} \mathrm{~F}$-hGTS13 radioactivity in the $\mathrm{H} 460$ tumor was determined to be $6.4 \pm 0.9 \% \mathrm{ID} / \mathrm{g}$, which was significantly higher than that of blood $(0.6 \pm 0.2 \% \mathrm{ID} / \mathrm{g}, P=0.0042, n=3)$ and muscle $(0.1 \pm 0.05 \%$ $\mathrm{ID} / \mathrm{g}, P=0.0004, n=3)$.

\section{Separation of ${ }^{18} \mathrm{~F}-\mathrm{hGTS} 13 \mathrm{C}-5$ Epimers and Evaluation in H460 Tumor-Bearing Rats}

Chiral high-performance liquid chromatography of compound 1 gave baseline separation of C-5 epimers with retention times of 6.4 and $8.5 \mathrm{~min}$. No efforts were made to resolve the absolute stereochemistry of the isomers at this stage. They were defined as isomer 1 and isomer 2 based on order of elution from the chiral
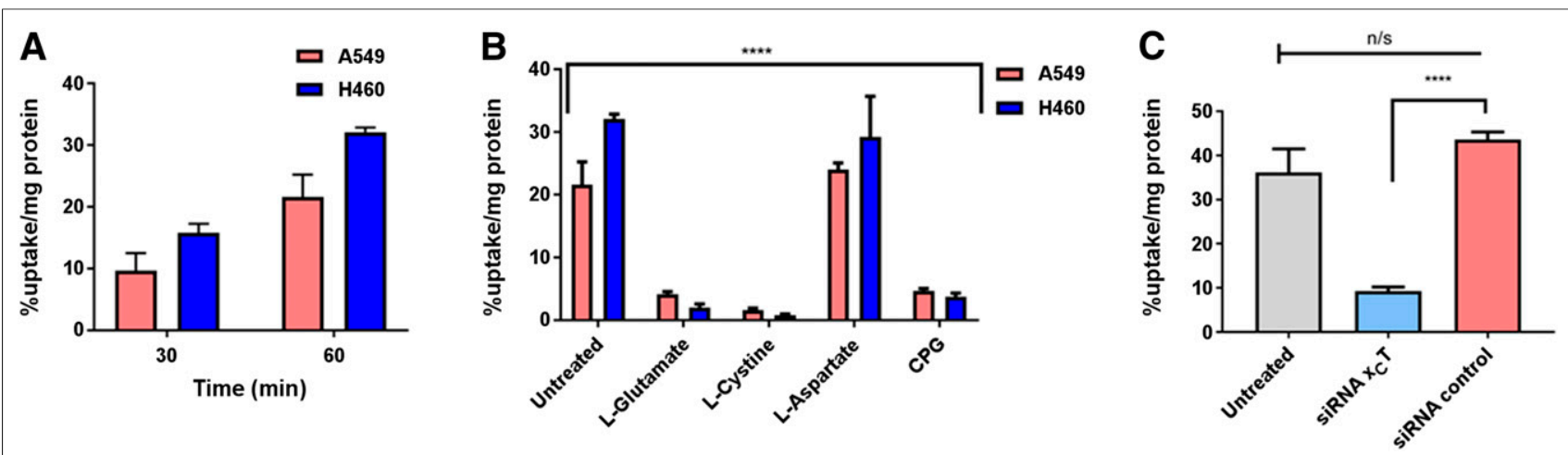

FIGURE 2. (A) Cellular uptake of ${ }^{18} \mathrm{~F}-\mathrm{hGTS} 13$ over time in $\mathrm{A} 549$ and $\mathrm{H} 460$ lung carcinoma cell lines. (B) Competition studies of ${ }^{18} \mathrm{~F}-\mathrm{hGTS} 13$ in $\mathrm{A} 549$ and $\mathrm{H} 460$ cells at $60 \mathrm{~min}$ after addition of radioactivity. There was significant interaction on inhibitor and cell line $\left({ }^{\star \star \star \star} P<0.0001, n=3\right.$ per condition). In A549 and H460 cells, ${ }^{18} \mathrm{~F}-\mathrm{hGTS} 13$ uptake was significantly reduced in L-glutamate-treated cells $(P<0.0001$ for both cell lines; A549, $81 \%$ decrease; H460, 94\% decrease), L-cystine-treated cells ( $P<0.0001$ for both cell lines; A549, 93\% decrease; H460, $97 \%$ decrease), and (S)-4carboxylphenylglycine (CPG)-treated cells $(P<0.0001$ for both cell lines; A549, $78 \%$ decrease; $\mathrm{H} 460$, $88 \%$ decrease). Nonsignificant reduction was observed in L-aspartate-treated cells $\left(P=0.74\right.$ for A549 cells, $P=0.59$ for $\mathrm{H} 460$ cells). (C) Reduced ${ }^{18} \mathrm{~F}$-hGTS13 uptake was evident in A549 cells treated with $\mathrm{x}_{C} T$ siRNA compared with control siRNA $(P<0.0001, n=3$ per condition). No difference in uptake was evident in untreated cells compared with control siRNA $(P=0.10, n=3$ per condition). 


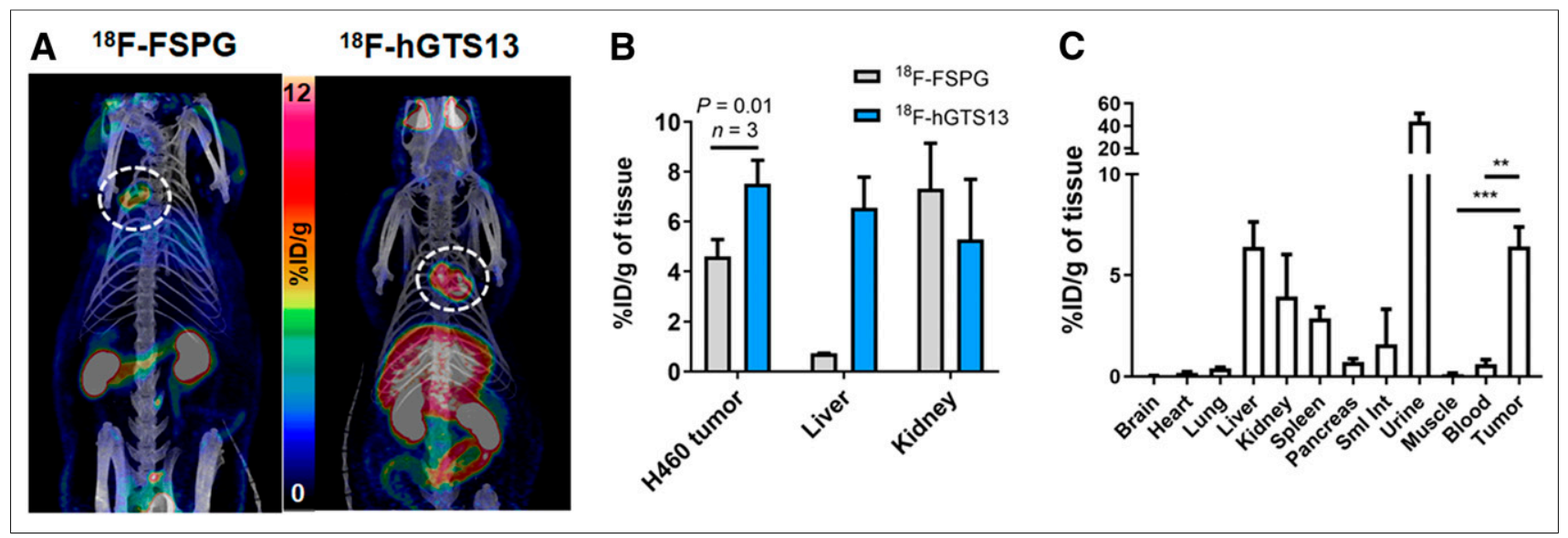

FIGURE 3. (A) Representative maximum-intensity-projection PET/CT images of rats bearing subcutaneous $\mathrm{H} 460$ tumors 60 min after intravenous administration of $\sim 15 \mathrm{MBq}$ of ${ }^{18} \mathrm{~F}-\mathrm{FSPG}$ or ${ }^{18} \mathrm{~F}$-hGTS13. (B) Levels of radioactivity in $\mathrm{H} 460$ tumors at 60 min after injection of radioactivity with ${ }^{18} \mathrm{~F}-$ FSPG and ${ }^{18} \mathrm{~F}-\mathrm{hGTS} 13(P=0.01, n=3)$ and in liver and kidneys determined from PET imaging. (C) Ex vivo biodistribution at $\sim 80$ min after injection of ${ }^{18} \mathrm{~F}$-hGTS13 in 3 animals. ${ }^{* *} P=0.0042 .{ }^{* \star} P=0.0004$. Sml Int $=$ small intestine.

column. Radiolabeling of the C-5 epimers of compound 1 to give ${ }^{18}$ F-hGTS13-isomer1 and ${ }^{18} \mathrm{~F}$-hGTS13-isomer2 was achieved using the method described for the racemic mixture. ${ }^{18} \mathrm{~F}-\mathrm{hGTS} 13$-isomer1 and ${ }^{18} \mathrm{~F}$-hGTS13-isomer2 were synthesized, respectively, in $4.4 \%$ $(n=1)$ and $5.1 \% \pm 1.7 \%(n=2)$ radiochemical yield, non-decaycorrected. H460 tumor-bearing rats were evaluated with static PET/ CT imaging of ${ }^{18} \mathrm{~F}$-hGTS13-isomer1 and ${ }^{18} \mathrm{~F}$-hGTS13-isomer2 (4 $\mathrm{d}$ apart) (Fig. 4). A significant reduction in liver uptake was observed with ${ }^{18} \mathrm{~F}$-hGTS13-isomer2 compared with ${ }^{18} \mathrm{~F}-\mathrm{hGTS} 13-$ isomer1 at $60 \mathrm{~min}$ after injection of radioactivity $(4.7 \pm 0.9$ vs. $8.5 \pm 0.4 \% \mathrm{ID} / \mathrm{g}, P=0.01, n=3$ ). There were similar amounts of radioactivity retained in $\mathrm{H} 460$ tumors between ${ }^{18} \mathrm{~F}$-hGTS13isomer 1 and ${ }^{18} \mathrm{~F}$-hGTS13-isomer2 $(6.7 \pm 1.1$ vs. $6.3 \pm 1.6 \%$ $\mathrm{ID} / \mathrm{g}, P=0.8, n=3)$. Further studies were completed with ${ }^{18}$ F-hGTS13-isomer2 based on the improved biodistribution profile and similar levels of radioactivity retained in H460 tumors. Dynamic PET/CT imaging with ${ }^{18} \mathrm{~F}$-hGTS13-isomer2 was completed over a 60-min period (Fig. 5). High H460 tumor accumulation was evident throughout the scan, with radiotracer uptake continuing to rise over the $60-\mathrm{min}$ period $(4.7 \pm 1.3 \% \mathrm{ID} / \mathrm{g}$ at $60 \mathrm{~min}$ after injection), suggesting that peak tumor radiotracer uptake may occur beyond the 60-min acquisition period. Levels of radioactivity present in healthy lung tissue and muscle were low throughout the scan, highlighting the potential for a high tumor-to-background ratio in cancers of the thorax (Supplemental Fig. 6A). Results of the PET imaging studies were confirmed through ex vivo biodistribution (Supplemental Fig. 6B).

\section{Evaluation of ${ }^{18} \mathrm{~F}-\mathrm{hGTS} 13-$ Isomer2 in Immune Cell Populations Compared with ${ }^{18}$ F-FSPG}

We evaluated the uptake of ${ }^{18} \mathrm{~F}-\mathrm{hGTS} 13$-isomer2 compared with ${ }^{18} \mathrm{~F}-\mathrm{FSPG}$ in primary human T cells, B cells, and monocytes, which are key immune cell populations in various inflammatory diseases and the tumor microenvironment. These studies revealed a dramatic increase in the uptake of ${ }^{18} \mathrm{~F}-\mathrm{FSPG}$ in activated versus resting $\mathrm{T}$ cells (72-fold, $P<0.0001, n=3$ ) (Fig. 6A), consistent with previous studies (26). In contrast, the increased uptake of ${ }^{18} \mathrm{~F}$ hGTS13-isomer2 in activated $\mathrm{T}$ cells was attenuated; there was a 17-fold increase relative to resting $\mathrm{T}$ cells $(P=0.0002, n=3)$. A similar trend was observed with $\mathrm{B}$ cells, with ${ }^{18} \mathrm{~F}-\mathrm{FSPG}$ exhibiting a 3-fold increased uptake in activated versus resting B cells $(P=0.0003, n=3$, Fig. 6B). In contrast, ${ }^{18} \mathrm{~F}$-hGTS13-isomer2 displayed a 2-fold increase in uptake in activated versus resting B cells $(P=0.189, n=3)$. Finally in monocytes, ${ }^{18} \mathrm{~F}$-FSPG displayed a 2 -fold increased uptake in activated versus resting monocytes $(P=0.0022, n=3)$ whereas ${ }^{18} \mathrm{~F}$-hGTS13-isomer2 exhibited a 4-fold increased uptake in activated versus resting monocytes ( $P<0.0001, n=3$, Fig. 6C). However, ${ }^{18}$ F-hGTS13-isomer2 absolute uptake values were considerably lower.

System $\mathrm{x}_{\mathrm{C}}{ }^{-}$represents an attractive target for PET imaging because of its involvement in mediating cellular response to oxidative stress and detoxification processes that support tumor progression and therapy
FIGURE 4. (A) Representative maximum-intensity-projection PET/CT images at 60 min after tailvein injection of $\sim 15 \mathrm{MBq}$ of ${ }^{18} \mathrm{~F}$-hGTS13-isomer1 or ${ }^{18} \mathrm{~F}$-hGTS13-isomer2 in $\mathrm{H} 460$ tumor-bearing rats. (B) Levels of radioactivity retained in $\mathrm{H} 460$ tumors and liver $(P=0.01, n=3)$.

\section{DISCUSSION}

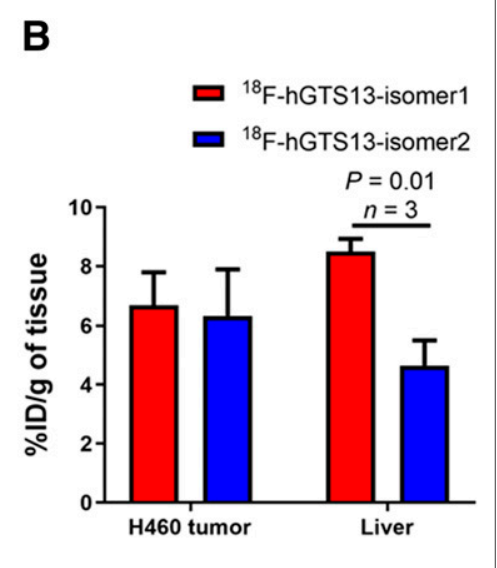

Characterization of ${ }^{18}$ F-hGTS13 • Beinat et al. 


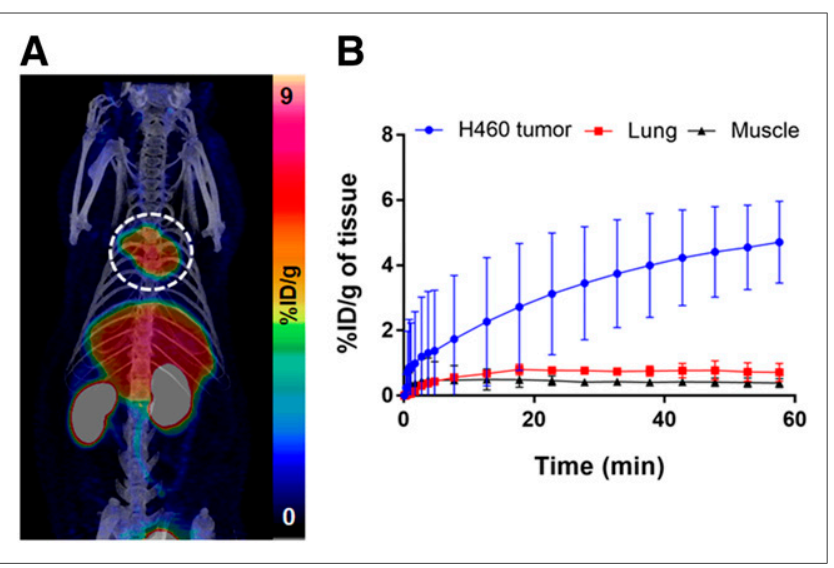

FIGURE 5. (A) Representative summed 25- to 60-min maximum-intensity-projection PET/CT image of $\mathrm{H} 460$ tumor-bearing rat. (B) Time-activity curves reflecting ${ }^{18} \mathrm{~F}$-hGTS13-isomer2 tumor uptake.

resistance. Molecular imaging of $\mathrm{x}_{\mathrm{C}}{ }^{-}$activity provides additional information over other amino acid-based PET probes currently being explored, including ${ }^{18}$ F-FET and ${ }^{18}$ F-FDOPA, which are taken up primarily by L-type amino acid transporter mechanisms and largely reflect nutrient uptake to support increased biomass and proliferative energy demands (28). The present work describes the characterization of a novel radiotracer, ${ }^{18} \mathrm{~F}-\mathrm{hGTS} 13$-isomer2, for PET imaging of $\mathrm{x}_{\mathrm{C}}{ }^{-}$ activity and evaluation in cell culture and preclinical models.

The capacity of cancer cells to achieve multidrug resistance remains an obstacle for successful chemotherapy. $\mathrm{x}_{\mathrm{C}}{ }^{-}$plays a vital role in the development of multidrug resistance in cancer. Increased $\mathrm{x}_{\mathrm{C}}{ }^{-}$expression is associated with chemoresistance of tumor cells; a negative correlation is evident between its expression and drug potency across NCI-60 cancer cell lines (29). A negative correlation is similarly evident between $\mathrm{x}_{\mathrm{C}}{ }^{-}$expression and the anticancer activity of compounds that contain structural features amenable to GSH reactivity, including Mannich base, therefore indicating that GSH-dependent inactivation is a potential mechanism through which $\mathrm{x}_{\mathrm{C}}{ }^{-}$expression induces chemoresistance (30). Molecular imaging of $\mathrm{x}_{\mathrm{C}}{ }^{-}$activity provides the potential to visualize this important target in oncology and gain insight into the mechanisms of cancer drug resistance. PET imaging of $\mathrm{x}_{\mathrm{C}}{ }^{-}$activity with ${ }^{18} \mathrm{~F}$-hGTS13-isomer2 may provide the potential to visualize tumors and provide valuable insight into chemoresistance, particularly in cancers of the thorax because of the low background signal in healthy tissue. Although ${ }^{18} \mathrm{~F}$-hGTS13-isomer2 displayed a favorable increase in tumor uptake, an increase in radiotracer uptake was also evident in the liver compared with ${ }^{18} \mathrm{~F}-\mathrm{FSPG}$, potentially limiting the ability of this radiotracer for imaging hepatocellular cancer, for which ${ }^{18} \mathrm{~F}-\mathrm{FSPG}$ has shown utility (31). There are no anticipated concerns in radiation dosimetry based on the increased liver uptake. Key applications in which ${ }^{18} \mathrm{~F}$-hGTS13-isomer2 may have advantages over ${ }^{18} \mathrm{~F}$ FSPG include lung cancer, breast cancer, and primary and metastatic brain lesions.

Several lines of evidence suggest the role of $\mathrm{x}_{\mathrm{C}}{ }^{-}$in regulating the innate and adaptive immune response. Activation of monocytes is associated with production of reactive oxygen species. The $\mathrm{x}_{\mathrm{C}}{ }^{-}$is subsequently upregulated potentially as an autoprotective response during activation of these cells (32). Similarly, naïve $\mathrm{T}$ cells are known to be metabolically dependent on antigen-presenting cells to fulfil their cysteine requirements due to the lack of $\mathrm{x}_{\mathrm{C}}{ }^{-}$and limited cysteine availability in the extracellular space. However, on activation of $\mathrm{T}$ cells, expression of $\mathrm{x}_{\mathrm{C}}{ }^{-}$becomes upregulated and provides activated $\mathrm{T}$ cells with cysteine necessary for proliferation $(33,34)$. Aside from malignant cells, the tumor microenvironment contains a complex milieu of immune cells including T- and B-lymphocytes, tumor-associated macrophages and monocytes, and fibroblasts and vascular endothelial cells (35). The $\mathrm{x}_{\mathrm{C}}{ }^{-}$expression and uptake of ${ }^{18} \mathrm{~F}-\mathrm{FSPG}$ in several of these cell populations have been previously investigated (26,33,36). Although ${ }^{18} \mathrm{~F}$-hGTS13isomer 2 uptake was elevated in activated versus resting immune cells, the differential observed between activated and resting cells, as well as the absolute levels of uptake, was much lower than observed with ${ }^{18}$ F-FSPG. Although both compounds are specifically taken up through the same transporter, structural differences (length of the carbon backbone and the side chain) may cause more directed inward channeling of one compound over the other depending on the context of the transporter activity, that is, cancer or inflammation, and associated driving forces. Future studies including immune cell blocking studies will be required to evaluate this in detail. Additional studies including in vivo inflammation models will be required to determine whether these findings persist in animal models. Nevertheless, the results of ${ }^{18} \mathrm{~F}$-hGTS13-isomer 2 in cell culture and in animal models are promising and highlight the potential of

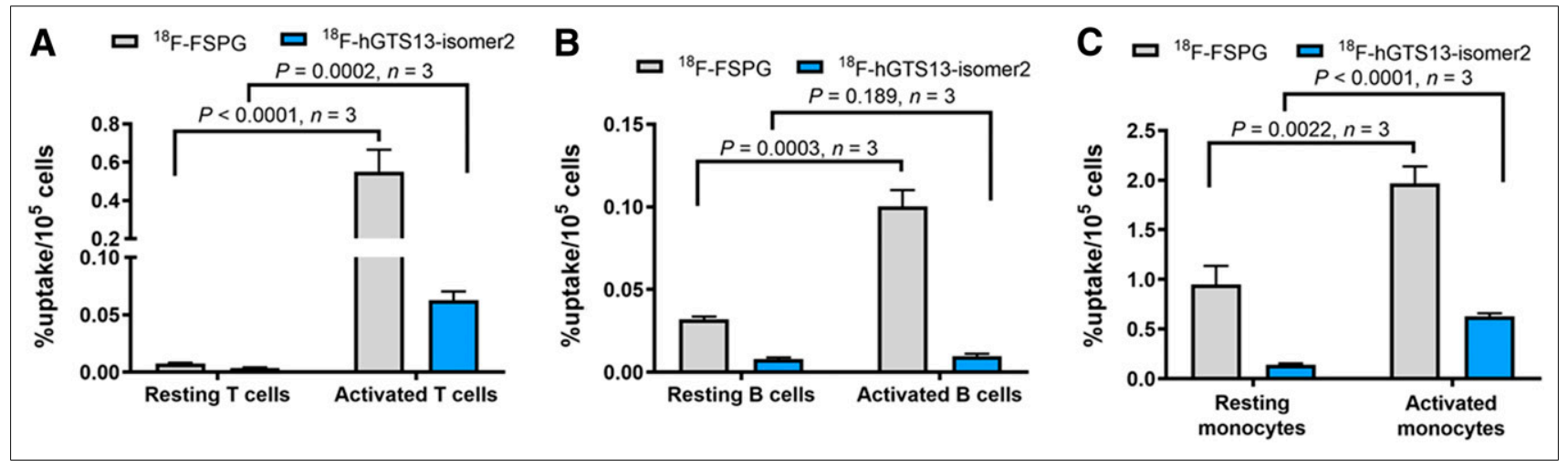

FIGURE 6. Cellular uptake of ${ }^{18} \mathrm{~F}-\mathrm{FSPG}$ and ${ }^{18} \mathrm{~F}$-hGTS13-isomer2 in resting and activated human T cells $\left({ }^{18} \mathrm{~F}-\mathrm{FSPG}\right.$ resting vs. activated, $P<0.0001$, $n=3 ;{ }^{18} \mathrm{~F}$-hGTS13-isomer2 resting vs. activated, $\left.P=0.0002, n=3\right)(\mathrm{A})$, B cells $\left({ }^{18} \mathrm{~F}-\mathrm{FSPG}\right.$ resting vs. activated, $P=0.0003, n=3 ;{ }^{18} \mathrm{~F}-\mathrm{hGTS} 13-$ isomer2 resting vs. activated, $P=0.189, n=3)(\mathrm{B})$, and monocytes $\left({ }^{18} \mathrm{~F}-\mathrm{FSPG}\right.$ resting vs. activated, $P=0.0022, n=3 ;{ }^{18} \mathrm{~F}$-hGTS13-isomer2 resting vs. activated, $P<0.0001, n=3)(\mathrm{C})$. 
this radiotracer with improved specificity for imaging cancer cells in the tumor microenvironment.

\section{CONCLUSION}

${ }^{18} \mathrm{~F}$-hGTS13-isomer2 is a new radiotracer for molecular imaging of $\mathrm{x}_{\mathrm{C}}{ }^{-}$activity, specifically taken up by $\mathrm{x}_{\mathrm{C}}{ }^{-}$in cell culture and with high tumor accumulation in animal models. Molecular imaging of $\mathrm{x}_{\mathrm{C}}{ }^{-}$activity with ${ }^{18} \mathrm{~F}$-hGTS13-isomer2 has potential for improved tumor detection and staging compared with other $\mathrm{x}_{\mathrm{C}}{ }^{-}$-targeting radiotracers and may assist in directing therapeutic strategies by visualizing metabolic adaptations to oxidative stress. Future studies are required to elucidate the uptake of ${ }^{18} \mathrm{~F}$-hGTS13-isomer2 in inflammation states in vivo and to associate radiotracer uptake with chemoresistance. Clinical translation of ${ }^{18} \mathrm{~F}$-hGTS13-isomer2 is under way for molecular imaging of cancers of the thorax.

\section{DISCLOSURE}

This work was partly supported by Life Molecular Imaging GmbH (formerly Piramal Imaging). Corinne Beinat acknowledges receipt of a Stanford Translational Research and Applied Medicine Fellowship. The authors acknowledge support from Thomas Brumby, Georg Kettschau, and Timo Stellfeld of Bayer Pharma (Berlin, Germany) in the development of this compound. No other potential conflict of interest relevant to this article was reported.

\section{ACKNOWLEDGMENTS}

We thank the Radiochemistry Facility and Small Animal Imaging Facility at Stanford University.

\section{KEY POINTS}

QUESTION: The goal of this study was development of a novel radiotracer for molecular imaging of $\mathrm{x}_{\mathrm{C}}{ }^{-}$activity with improved tumor uptake and reduced uptake in inflammatory cells.

PERTINENT FINDINGS: 18 F-hGTS13-isomer2 exhibited excellent $\mathrm{H} 460$ tumor visualization and significantly reduced uptake in multiple immune cell populations.

IMPLICATIONS FOR PATIENT CARE: ${ }^{18} \mathrm{~F}$-hGTS13-isomer2 has potential for clinical translation for imaging cancers of the thorax because of the low background signal in healthy tissue.

\section{REFERENCES}

1. Kelloff GJ, Hoffman JM, Johnson B, et al. Progress and promise of FDG-PET imaging for cancer patient management and oncologic drug development. Clin Cancer Res. 2005;11:2785-2808.

2. Pantel AR, Ackerman D, Lee SC, Mankoff DA, Gade TP. Imaging cancer metabolism: underlying biology and emerging strategies. J Nucl Med. 2018;59: 1340-1349.

3. Shreve PD, Anzai Y, Wahl RL. Pitfalls in oncologic diagnosis with FDG PET imaging: physiologic and benign variants. Radiographics. 1999;19:61-77.

4. Mankoff DA, Eary JF, Link JM, et al. Tumor-specific positron emission tomography imaging in patients: $\left[{ }^{18} \mathrm{~F}\right]$ fluorodeoxyglucose and beyond. Clin Cancer Res. 2007;13:3460-3469.

5. Levine AJ, Puzio-Kuter AM. The control of the metabolic switch in cancers by oncogenes and tumor suppressor genes. Science. 2010;330:1340-1344.

6. Okuno S, Sato H, Kuriyama-Matsumura K, et al. Role of cystine transport in intracellular glutathione level and cisplatin resistance in human ovarian cancer cell lines. Br J Cancer. 2003;88:951-956.

7. Meister A. Glutathione metabolism. Methods Enzymol. 1995;251:3-7.

8. DeBerardinis RJ, Cheng T. Q's next: the diverse functions of glutamine in metabolism, cell biology and cancer. Oncogene. 2010;29:313-324.
9. Estrela JM, Ortega A, Obrador E. Glutathione in cancer biology and therapy. Crit Rev Clin Lab Sci. 2006;43:143-181.

10. Lu SC. Glutathione synthesis. Biochim Biophys Acta. 2013;1830:3143-3153.

11. Bridges RJNN, Patel SA. System $\mathrm{xc}^{-}$cystine/glutamate antiporter: an update on molecular pharmacology and roles within the cns. Br J Pharmacol. 2012;165:20-34.

12. Bassi MT, Gasol E, Manzoni M, et al. Identification and characterisation of human xct that co-expresses, with $4 \mathrm{f} 2$ heavy chain, the amino acid transport activity system xc. Pflugers Arch. 2001;442:286-296.

13. Patel SA, Warren BA, Rhoderick JF, Bridges RJ. Differentiation of substrate and non-substrate inhibitors of transport system $\mathrm{xc}^{-}$: an obligate exchanger of L-glutamate and L-cystine. Neuropharmacology. 2004;46:273-284.

14. Baek S, Choi CM, Ahn SH, et al. Exploratory clinical trial of $(4 \mathrm{~s})-4-\left(3-\left[{ }^{18} \mathrm{~F}\right]\right.$ fluoropropyl)-L-glutamate for imaging $\mathrm{xc}^{-}$transporter using positron emission tomography in patients with non-small cell lung or breast cancer. Clin Cancer Res. 2012;18:5427-5437.

15. Baek S, Mueller A, Lim Y-S, et al. (4s)-4-(3- ${ }^{18}$ F-fluoropropyl)-L-glutamate for imaging of $\mathrm{xc}^{-}$transporter activity in hepatocellular carcinoma using pet: preclinical and exploratory clinical studies. J Nucl Med. 2013;54:117-123.

16. Mittra ES, Koglin N, Mosci C, et al. Pilot preclinical and clinical evaluation of (4S)-4-(3-[ $\left[{ }^{18} \mathrm{~F}\right]$ fluoropropyl)-L-glutamate $\left({ }^{18} \mathrm{~F}-\mathrm{FSPG}\right)$ for PET/CT imaging of intracranial malignancies.PLoS One. 2016;11:e0148628.

17. Webster JM, Morton CA, Johnson BF, et al. Functional imaging of oxidative stress with a novel PET imaging agent, ${ }^{18} \mathrm{~F}$-5-fluoro-L-aminosuberic acid. $\mathrm{J} \mathrm{Nucl}$ Med. 2014;55:657-664.

18. Yang H, Jenni S, Colovic M, et al. ${ }^{18} \mathrm{~F}-5$-fluoroaminosuberic acid as a potential tracer to gauge oxidative stress in breast cancer models. J Nucl Med. 2017;58:367-373.

19. Chae SY, Choi CM, Shim TS, et al. Exploratory clinical investigation of $(4 S)$-4(3- ${ }^{18}$ F-fluoropropyl)-L-glutamate PET of inflammatory and infectious lesions. J Nucl Med. 2016;57:67-69.

20. Martín A, Vázquez-Villoldo N, Gómez-Vallejo V, et al. In vivo imaging of system $\mathrm{xc}^{-}$as a novel approach to monitor multiple sclerosis. Eur J Nucl Med Mol Imaging. 2016;43:1124-1138.

21. Domercq M, Szczupak B, Gejo J, et al. PET imaging with $\left[{ }^{18} \mathrm{~F}\right] \mathrm{FSPG}$ evidences the role of system $\mathrm{xc}^{-}$on brain inflammation following cerebral ischemia in rats. Theranostics. 2016;6:1753-1767.

22. Beinat C, Haywood T, Chen YS, et al. The utility of $\left[{ }^{18} \mathrm{~F}\right] \mathrm{DASA}-23$ for molecular imaging of prostate cancer with positron emission tomography. Mol Imaging Biol. 2018;20:1015-1024.

23. Krieg AM, Yi A-K, Matson S, et al. Cpg motifs in bacterial DNA trigger direct b-cell activation. Nature. 1995;374:546-549.

24. Trickett A, Kwan YL. T cell stimulation and expansion using anti-cd3/cd28 beads. J Immunol Methods. 2003;275:251-255.

25. Plevin RE, Knoll M, McKay M, Arbabi S, Cuschieri J. The role of lipopolysaccharide structure in monocyte activation and cytokine secretion. Shock. 2016;45:22-27.

26. Hoehne A, James ML, Alam IS, et al. [ $\left.{ }^{18} \mathrm{~F}\right]$ FSPG-PET reveals increased cystine/ glutamate antiporter $\left(\mathrm{xc}^{-}\right)$activity in a mouse model of multiple sclerosis. J Neuroinflammation. 2018;15:55.

27. Koglin N, Mueller A, Berndt M, et al. Specific PET imaging of xc transporter activity using a ${ }^{18} \mathrm{~F}$-labeled glutamate derivative reveals a dominant pathway in tumor metabolism. Clin Cancer Res. 2011;17:6000-6011.

28. Sun A, Liu X, Tang G. Carbon-11 and fluorine-18 labeled amino acid tracers for positron emission tomography imaging of tumors. Front Chem. 2018;5: 124.

29. Huang Y, Dai Z, Barbacioru C, Sadée W. Cystine-glutamate transporter slc7a11; in cancer chemosensitivity and chemoresistance. Cancer Res. 2005;65:7446-7454.

30. Dai Z, Huang Y, Sadee W, Blower P. Chemoinformatics analysis identifies cytotoxic compounds susceptible to chemoresistance mediated by glutathione and cystine/glutamate transport system xc. J Med Chem. 2007;50:1896-1906.

31. Kavanaugh G, Williams J, Morris AS, et al. Utility of $\left[{ }^{18} \mathrm{~F}\right] \mathrm{FSPG}$ PET to image hepatocellular carcinoma: first clinical evaluation in a US population. Mol Imaging Biol. 2016;18:924-934.

32. Pampliega O, Domercq M, Soria FN, Villoslada P, Rodríguez-Antigüedad A, Matute C. Increased expression of cystine/glutamate antiporter in multiple sclerosis. J Neuroinflammation. 2011;8:63.

33. Levring TB, Hansen AK, Nielsen BL, et al. Activated human CD4+ T cells express transporters for both cysteine and cystine. Sci Rep. 2012;2:266.

34. Siska PJ, Kim B, Ji X, et al. Fluorescence-based measurement of cystine uptake through xCT shows requirement for ROS detoxification in activated lymphocytes. J Immunol Methods. 2016;438:51-58.

35. Balkwill FR, Capasso M, Hagemann T. The tumor microenvironment at a glance. J Cell Sci. 2012;125:5591-5596.

36. Merckx E, Albertini G, Paterka M, et al. Absence of system $\mathrm{x}_{\mathrm{c}}{ }^{-}$on immune cells invading the central nervous system alleviates experimental autoimmune encephalitis. J Neuroinflammation. 2017;14:9. 